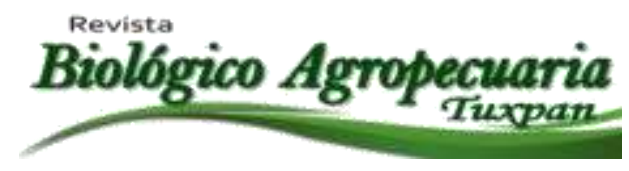

\title{
Helmintos intestinales de algunas aves acuáticas en el estado de Hidalgo: estudio de caso, Lago de Tecocomulco
}

Intestinal helminths of some waterfowl in the state of Hidalgo: case study, Lago of Tecocomulco Alemán-Canales Emmanuel ${ }^{\bowtie}$, Scott Monks y Griselda Pulido-Flores

Área Académica de Biología, Universidad Autónoma del Estado de Hidalgo, Carr. PachucaTulancingo Km. 4.5, Mineral de la Reforma, Hidalgo, México. C.P. 42184. Tel: (771)717-2000 ext. 6658. Fax: (771)717-2112.

${ }^{凶}$ Autor para correspondencia: alemancanemma@yahoo.com.mx

Recibido: 04/01/2014

Aceptado: 10/07/2014

\section{RESUMEN}

Como parte de un estudio de la calidad ambiental de Lago de Tecocomulco, Hidalgo, México, fueron colectados los helmintos intestinales de 70 aves de cinco especies: Anas americana, Anas discors, Aythya valisineria, Gallinula chloropus y Fulica americana. Los intestinos de las aves fueron donados por los cazadores locales, transportadas al laboratorio y los helmintos fueron colectados, fijados en AFA y conservados en alcohol etílico (70\%) hasta su tinción y montaje. Ocho especies de dos taxa de helmintos fueron encontrados: Digenea-Echinostoma revolutum, Zygocotyle lunata, Echinoparyphium recurvatum, Notocotylus pacifer, Apatemon gracilis, Pseudoapatemon sp.; y AcanthocephalaPolymorphus trochus y Pseudocorynosoma constrictum. Echinostomum revolutum tuvo la mayor prevalencia, abundancia e intensidad promedio en las 36 aves de la Familia Anatidae; una prevalencia de $40 \%$ fue encontrada en cada especie de ave de esta familia. Sin embargo, en $F$. americana (Rallidae), P. trochus tuvo la mayor prevalencia (58.6\%), una abundancia de 2.0 y $N$. pacifer tuvo mayor intensidad promedio (4.3). Echinostomum revolutum y Ps. Constrictum se registran por segunda ocasión en Hidalgo; Z. lunata, N. pacifer, A. gracilis y P. trochus son nuevos registros para las aves en Hidalgo y Pseudoapatemon sp. es un nuevo registro para México. Es importante monitorear los parásitos de las aves acuáticas migratorias en Lago de Tecocomulco ya que sirven como indicadores de la calidad ambiental del lago.

Palabras clave: Helmintos parásitos, Hidalgo, Aves acuáticas, Lago de Tecocomulco.

\begin{abstract}
As part of a study of the environmental quality of Lago de Tecocomulco, Hidalgo, México, intestinal helminths of waterfowl were collected from 70 birds of five species: Anas americana, Anas discors, Aythya valisineria, Gallinula chloropus and Fulica americana. Intestines of birds were provided by
\end{abstract}


local hunters, transported to the laboratory, and helminths were collected from each, fixed in AFA, and stored in Ethyl Alcohol (70\%) until stained and mounted on slides. Eight species of two taxa of helminth were found: Digenea- Echinostoma revolutum, Zygocotyle lunata, Echinoparyphium recurvatum, Notocotylus pacifer, Apatemon gracilis, Pseudoapatemon sp.; and AcanthocephalaPolymorphus trochus y Pseudocorynosoma constrictum. Echinostomum revolutum had the highest prevalence, abundance and mean intensity in the 36 birds of the Family Anatidae; a prevalence of $40 \%$ was found in each species of bird. However, in $F$. americana (Rallidae), the acanthocephalan $P$. trochus had a higher prevalence $(58.6 \%$ ) and an abundance of 2.0, and $N$. pacifer had a higher mean intensity (4.3). Echinostomum revolutum and Ps. constrictum are recorded only for the second time in Hidalgo; Z. lunata, N. pacifer, A. gracilis and P. trochus are new records for birds in Hidalgo, and Pseudoapatemon sp. is a new record for Mexico. It is important to monitor the parasites of the migratory waterfowl visiting Lago de Tecocomulco as an indicator of the environmental quality of the lake.

Keywords: Helminth parasites, Hidalgo, waterfowl, Lake Tecocomulco.

\section{INTRODUCCIÓN}

Las aves acuáticas constituyen uno de los componentes más importantes de la fauna que habita los humedales. Las aves migratorias pueden hacer uso de estos ambientes durante sólo parte del año y para cubrir una determinada etapa de su ciclo anual. En estos humedales las aves acuáticas cumplen importantes roles como el de consumidores,

aportadores de materia orgánica y modificadores del ambiente circundante (Blanco y Canevari, 1995).

Entre los problemas que afectan a las aves silvestres, las enfermedades parasitarias se destacan como uno de los más frecuentes que en ocasiones muy severas puede causar la muerte de muchas de ellas (Figueroa-Lyra et al. 2002). Los estudios de parásitos de aves, son de gran interés e importancia debido a que estas presentan un mecanismo muy importante de dispersión de agentes patógenos en diferentes áreas geográficas (Barrera-Guzmán y GuillénHernández, 2008). Factores como la migración obligada de las aves acuáticas, unidas al cambio climático y su régimen alimenticio, principalmente a base de invertebrados en algunas especies, han incrementado la posibilidad de infección con los helmintos (Díaz et al. 2000).

Lago de Tecocomulco es el último humedal relicto que queda de la gran Cuenca de México y es un sitio importante para la conservación de la biodiversidad por lo que se cataloga como un sitio Ramsar (Convención Relativa a los Humedales de Importancia Internacional, Ramsar, Irán) y como un Área Importante para la Conservación de las Aves (AICA) (Bautista-Hernández et al. 2008). El objetivo de este trabajo fue conocer los helmintos intestinales de algunas aves acuáticas en el estado de Hidalgo, mediante colecta directa y revisión bibliográfica para contribuir al conocimiento de su helmintofauna.

\section{MATERIALES Y MÉTODOS}

El lago se ubica en la parte sureste del Estado de Hidalgo con coordenadas geográficas 19'42'14', y 19'59'30' de latitud norte y 98 $11^{\circ} 46^{\prime}$ ' y 98 $27^{\circ} 30^{\prime \prime}$ ' de longitud oeste (Jiménez-Fernández et al. 1996; HuízarÁlvarez et al. 2005; Bautista-Hernández et al. 2008). Durante los años 2006, 2007 y 2011 cazadores locales donaron intestinos de 70 aves de cinco especies (Anas americana, Anas discors, Aythya valisineria, Fulica americana y 
Gallinula chloropus) de Lago de Tecocomulco identificadas por nombre común. Se obtuvieron los órganos viscerales y se depositaron en bolsas herméticas etiquetadas para su transporte en una hielera.

Se extrajeron los helmintos en el laboratorio y se limpiaron, se fijaron con una solución a base de alcohol, formol y ácido acético (AFA; Pritchard y Kruse, 1982) y se conservaron en alcohol etílico al $70 \%$. Posteriormente, se realizó la tinción y montaje con técnicas convencionales en helmintología (Pritchard y Kruse, 1982). Las medidas se tomaron usando un ocular micrométrico calibrado y para la identificación se realizó de acuerdo con claves taxonómicas (McDonald, 1981; Yamaguti, 1971). Para caracterizar la infección de cada especie de ave, se calcularon los parámetros ecológicos recomendados por Margolis et al. (1982) y Bush et al. (1997).
Un total de 306 helmintos de las especies Echinostoma revolutum, Zygocotyle lunata, Echinoparyphium recurvatum, Notocotylus pacifer, Apatemon gracilis, Pseudoapatemon sp., Polymorphus trochus y Pseudocorynosoma constrictum fueron colectados de los intestinos de las aves. Echinostoma revolutum tuvo la mayor prevalencia, abundancia e intensidad promedio en las 36 aves revisadas de las dos especies de la familia Anatidae, destaca la prevalencia de este helminto en ambas especies con $40 \%$. En $F$. americana, $P$. trochus tuvo mayor prevalencia y abundancia $(58.6 \%$ y 2.0 , respectivamente) pero $N$. pacifer tuvo mayor intensidad promedio (4.3). En Ay. valisineria solo se encontró $Z$. lunata, con $33.3 \%$ de prevalencia, 0.33 de abundancia y 1.0 de intensidad promedio (Cuadro 1). Gallinula chloropus fue negativa a infección por endohelmintos.

\section{RESULTADOS Y DISCUSIÓN}

Cuadro 1. Helmintos intestinales de aves acuáticas de lago de Tecocomulco, Hidalgo, México. (NC=Nombre Común; E/P=Aves Examinadas/parasitadas; H=Especie de Helminto; P=Prevalencia; $\mathrm{A}=$ Abundancia; $\mathrm{I}=$ Intensidad promedio).

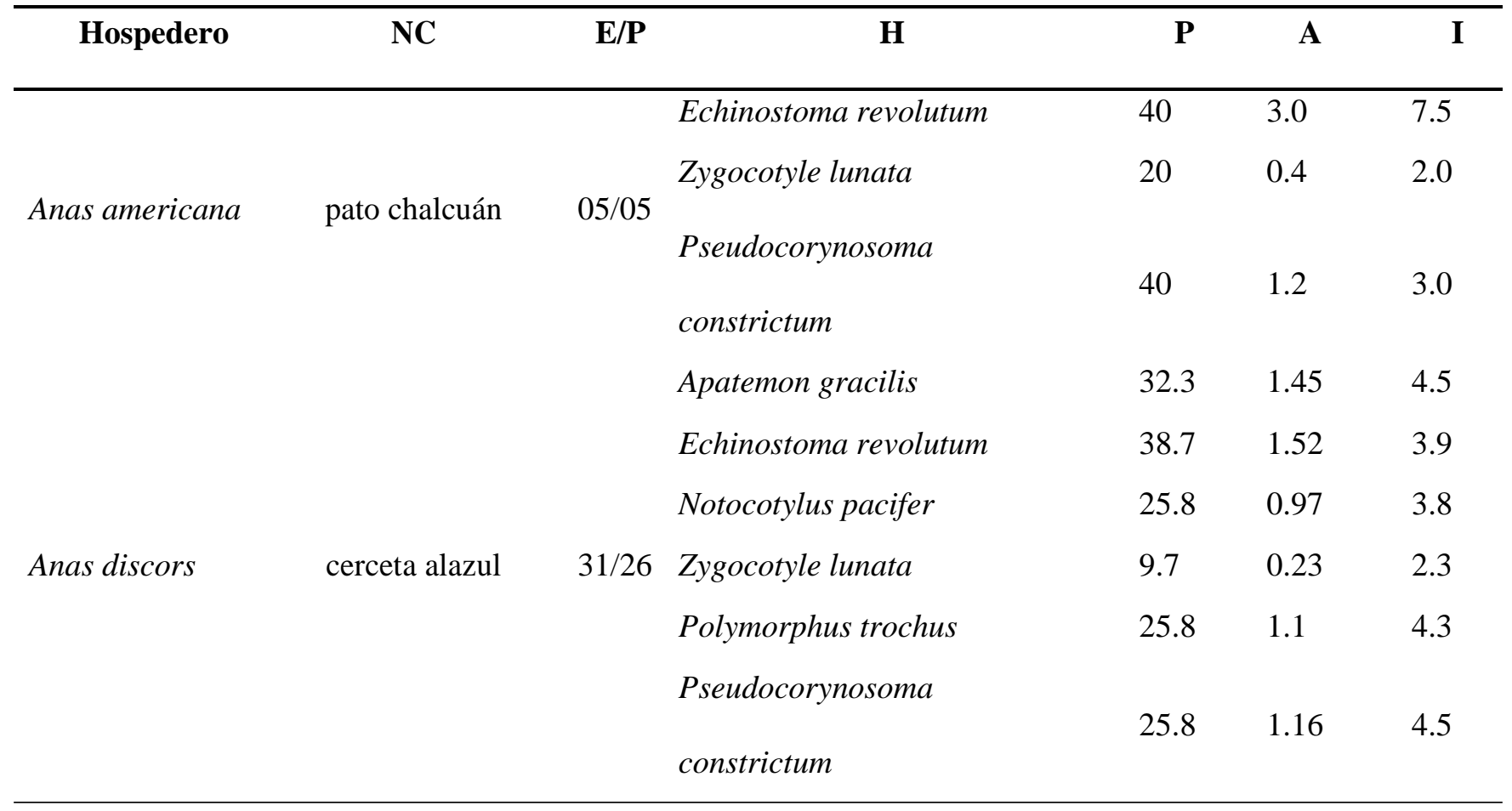




\begin{tabular}{lllllll}
\hline \multirow{2}{*}{ Aythya valisineria } & pato & \multirow{2}{*}{$03 / 01$} & Zygocotyle lunata & 33.3 & 0.33 & 1.0 \\
& coacoxtle & & & & & \\
& & & Pseudoapatemon sp. & 3.4 & 0.03 & 1.0 \\
& & & Echinostoma revolutum & 6.9 & 0.07 & 1.0 \\
\multirow{3}{*}{ Fulica americana } & gallareta & $29 / 28$ & Echinoparyphium recurvatum & 20.7 & 0.31 & 1.5 \\
& americana & & Notocotylus pacifer & 10.3 & 0.45 & 4.3 \\
& & & Polymorphus trochus & 58.6 & 2.0 & 3.4 \\
& & & & & & \\
& & & &
\end{tabular}

Adicional a esta colecta se incluyeron para Hidalgo los reportes de los helmintos $E$. revolutum, Cloacotaenia megalops y $P s$. constrictum en A. discors y el Nematoda Capillaria sp. en Oxyura jamaicensis datos del estudio de Padilla-Agular (2010), quien realizó un reporte de la helmintofauna de aves acuáticas del Altiplano Central Mexicano. Cabe mencionar que los únicos reportes de helmintofauna en aves de Hidalgo además de Padilla-Agular (2010), son los de Caballero y Peregrina (1938), quienes reportaron al nematodo Habronema mansoni en Buteo jamaicensis borealis y Alencaster (1948) quien reportó al nemátodo Aproctella nuda en Phainopepla nitens; ambos reportes son de aves terrestres del municipio de Tasquillo, Hidalgo.

Las especies $E$.revolutum y $P S$. constrictum fueron encontradas en el estudio Padilla-Aguilar (2010) y el presente trabajo; sin embargo, reportamos por primera vez las especies Z. lunata, $N$. pacifera, A. gracilis y $P$. trochas, como nuevos registros para Hidalgo y a Pseudoapatemon sp. como nuevo registro para México.

\section{CONCLUSIONES}

El estudio de parásitos de aves en el estado de Hidalgo, específicamente en humedales como Lago de Tecocomulco, tiene una gran importancia económica ya que estas aves son una fuente de ingresos para los habitantes de la zona ya que se ofrece como alimento en restaurantes que se encuentran a orillas del lago. Sin embargo, el estudio de estas aves también tiene una importancia científica. En primer lugar, porque son un componente de la biodiversidad, la conservación de estas especies es fundamental para el equilibrio de las comunidades en este y otros sitios de donde migran las aves. En segundo lugar, porque los

helmintos de aves funcionan como bioindicadores de la calidad ambiental del lago, es importante monitorearlos como una de las estrategias para mitigar el aumento de contaminantes. Finalmente, este estudio funciona como primer paso en el conocimiento y estudio de los ciclos de vida de los helmintos y con ello conocer parte de la biodiversidad en éste ecosistema, tan importante para el estado de Hidalgo y el país.

\section{LITERATURA CITADA}

Alencaster, I. G. 1948. Estudio monográfico de nematodos parásitos de las aves de México. Tesis Facultad de Ciencias, UNAM, México. 57 p.

Barrera-Guzmán, A. O. y Guillén-Hernández, S. 2008. Helmintos intestinales en aves Ciconiiformes de la Ciénega de Chuburná, Yucatán, México. Revista Mexicana de Biodiversidad 79:525-527. https://doi.org/10.22201/ib.20078706e.2008.002.54 9

Revista Científica Biológico Agropecuaria Tuxpan 2 (1)

ISSN: 2007-6940 
Bautista-Hernández, C. E., HernándezHernández, D. L., Monks, S. y PulidoFlores, G. 2008. Lago de Tecocomulco. In: G. Pulido-Flores, S. Monks, R. Miranda y D. Galicia (EDS). Estudios científicos del Lago Tecocomulco, Hidalgo, y zonas aledañas. Universidad Autónoma del Estado de Hidalgo y Universidad de Navarra (España), Pachuca, Hidalgo. p. 15-22.

https://doi.org/10.21041/conpat2019/v3rec232

Blanco, D. E. y Canevari, P. 1995. Situación actual de los chorlos y playeros migratorios de la Zona Costera Patagónica (provincias de Río Negro, Chubut y Santa Cruz). Humedales para las Américas. PMIZCP: Informe Técnico No. 3 (FPN-GEF-PNUDWCS), $26 \mathrm{p}$. https://doi.org/10.35537/10915/57176

Bush, A. O., Kevin D. L., Jeffrey M. L. y Shostak, A. W. 1997. Parasitology meets ecology on its own terms: Margolis et al. revisited. Journal of Parasitology 83:575-583. https://doi.org/10.2307/3284227

Caballero, C. E. y Peregrina, D. I. 1938. Contribución al conocimiento de los nematodos de las aves de México. VII. Anales del Instituto de Biología de la Universidad Nacional Autónoma de México 9:289-306. https://doi.org/10.29104/phi-aqualac/2010-v2-2-03

Díaz, V., Mañas, I., Campos, M. y Lozano, J. 2000. Contribución al conocimiento de los trematodos parásitos de túrdidoszorzales- en Granada, España. ARS Pharmaceutica 41:379-381.

Figueroa-Lyra, M., Bianque-De Oliveira, J., De Brito-Cavalcanti, M. D., SoaresLeite, A., Santiago-Magalhaes, V., Alves-De Oliveira, R. y EvencioSobrino, A. 2002. Parásitos gastrointestinales de aves silvestres en cautiverio en el estado de Pernambuco, Brasil. Parasitología latinoamericana 57:50-54.

https://doi.org/10.4067/s0717-77122002000100012

Huízar-Álvarez, R. y Ruíz-González, 2005

Aspectos físicos y socioeconómicos de la subcuenca de Tecocomulco. In: R.
Huizar-Álvarez, E. J. Jiménez-

Fernández y C. Juárez-López (EDS). La Laguna de Tecocomulco, Geo-ecología de un desastre, Instituto de Geología,

México, D. F. pp. 9-20.

Jiménez-Fernández, E. J., Juárez-López, J. C. y Cedillo-Álvarez, C. 1996. Guía para la tercera expedición del taller de fauna silvestre y acuática del Neoártico y https://doi.org/10.22201/fi.25940732e.2007 $.08 \mathrm{n} 2.007$

Neotrópico a la Laguna de Tecocomulco, Hidalgo. Universidad Nacional Autónoma de México, México, D. F. 46 p.

Margolis, L., Esch, G. W., Holmes, J. C., Kuris, A. M. y Schad, G. A. 1982. The use of ecological terms in parasitology (Report of an ad hoc committee of The American Society of Parasitologists). Journal of Parasitology 68:131-133. https://doi.org/10.2307/3281335

McDonald, M. E. 1981. Key to trematodes reported in waterfowl. Washington, D. C., United States Department of the Interior Fish and Wildlife Service/ Resource Publication, $142 \mathrm{p}$.

Padilla-Aguilar, P. 2010. Identificación de helmintofauna del tracto digestivo de aves acuáticas del altiplano central mexicano. Tesis de Licenciatura. Universidad Autónoma del Estado de Hidalgo, México. $97 \mathrm{p}$. https://doi.org/10.22201/ib.9786073020305e. 2019.c19

Pritchard, M. H. y Kruse, G. O. W. 1982. The collection and preservation of animal parasites. University of Nebraska Press, Lincoln, Nebraska, $141 \mathrm{p}$.

Yamaguti, S. 1971. Synopsis of digenetic trematodes of vertebrates. Tokyo, Japan, Keigaku Publishing Company, $1074 \mathrm{p}$. 


\section{Copyright (c) 2014 Emmamuel Alemán C anales, Scott Monks y Griseld a Pulido Flores}

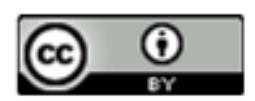

Este texto está protegido por una licencia Creative Common 4.0 .

Usted es libre para Compartir —copiar y redistribuir el material en cualquier medio o formato- y Ad aptar el documento —remezclar, transformar y crear a partir del material- para cualquier propósito, inchso para fines comerciales, siempre que cumpla la condición de:

Atribución: Usted debe dar crédito a la obra original de manera adecuada, proporcionar un enlace a la licencia, e indicar si se han realizado cambios. Puede hacerlo en cualquier forma razonable, pero no de forma tal que sugiera que tiene el apoyo del licenciante olo recibe por el usoque hace de la obra.

Resumende licencia - Textocompletodelalicenria 